\title{
The US Muon Accelerator Program (MAP)
}

\author{
Alan D. Bross ${ }^{1}$ \\ Fermi National Accelerator Laboratory, P.O. Box 500, Batavia, IL 60510 USA
}

\begin{abstract}
The US Department of Energy Office of High Energy Physics has recently approved a Muon Accelerator Program (MAP). The primary goal of this effort is to deliver a Design Feasibility Study for a Muon Collider after a 7 year R\&D program. This paper presents a brief physics motivation for, and the description of, a Muon Collider facility and then gives an overview of the program. I will then describe in some detail the primary components of the effort.
\end{abstract}

Keywords: muon collider, neutrino factory

PACS: $29.20 .-\mathrm{c}$

\section{PHYSICS AT A MUON ACCELERATOR FACILITY}

The physics potential of a high-energy lepton collider has been understudy for many years [1] [2] and there is a strong case to made that in order to fully exploit new physics discoveries at the LHC, a mult-TeV lepton collider will be needed. A multi-TeV lepton collider will not only allow experimenters to access higher mass supersymmetric particles, but can shed detailed insight into the understanding of the mechanism behind mass generation and electroweak symmetry breaking, while reaching beyond the capabilities of the LHC with respect to the search for evidence for extra dimensions or quantum gravity. In addition, the ability to make precision measurements of standard model processes will open windows on physics at energy scales beyond our direct reach. An example of the extended reach of a multi-TeV lepton collider comes from the 2004 CLIC physics study [3], Fig. 1. This figure illustrates the number of supersymmetric particles observable at various colliders (the $\mathrm{LHC}+e^{+} e^{-}$machines with center of mass energy up to $3 \mathrm{TeV}$ ) under a number of benchmark supersymmetric scenarios. The extended reach of a multi-TeV lepton collider is clear.

The Muon Collider (MC) provides a possible realization of a multi-TeV lepton collider, and hence a way to explore territory beyond the reach of the LHC. A muon accelerator facility also presents the opportunity to explore new physics within in a number of programs. A schematic that shows the evolution of a muon accelerator complex which ultimately reaches a multi-TeV Muon Collider [4] is shown schematically in Fig. 2. The front-end of the facility provides an intense muon source that can perhaps support both an energy-frontier

\footnotetext{
${ }^{1}$ For the Neutrino Factory and Muon Collider Collaboration
}

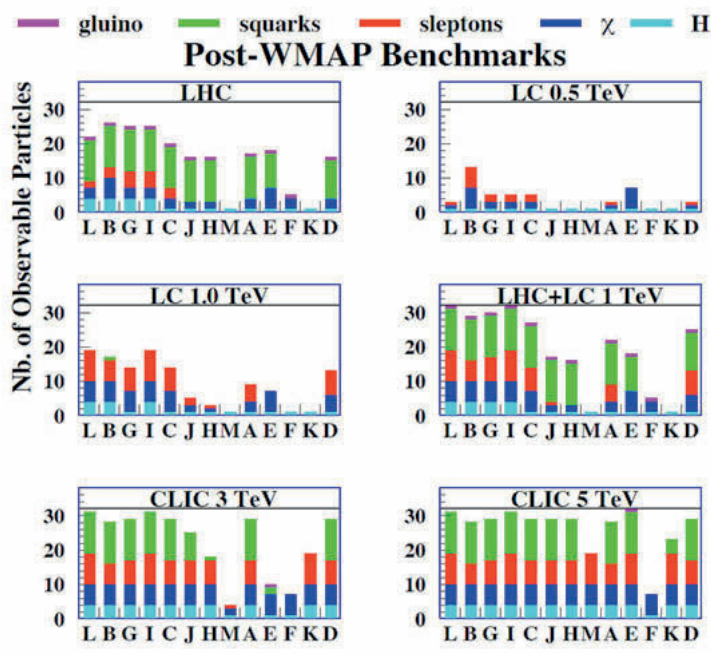

FIGURE 1. Bar graphs of the number of supersymmetric particles observable at different colliders and under different supersymmetry scenarios. See [3] for details.

Muon Collider and a Neutrino Factory. The muon source is designed to deliver $\mathscr{O} 10^{21}$ low energy muons per year within the acceptance of the accelerator system, and consists of (i) a multi-MW proton source delivering a multi$\mathrm{GeV}$ proton beam onto a liquid Mercury pion production target, (ii) a high-field target solenoid that radially confines the secondary charged pions, (iii) a long solenoidal channel in which the pions decay to produce positive and negative muons, (iv) a system of RF cavities in a solenoidal channel that capture the muons in bunches and reduce their energy spread (phase rotation), and (v) a muon ionization cooling channel that reduces the transverse phase space occupied by the beam by a factor of a few in each transverse direction. At this point the beam will fit within the acceptance of an accelerator for a Neutrino Factory. However, to obtain sufficient luminosity, a Muon Collider requires a great deal more muon cool- 


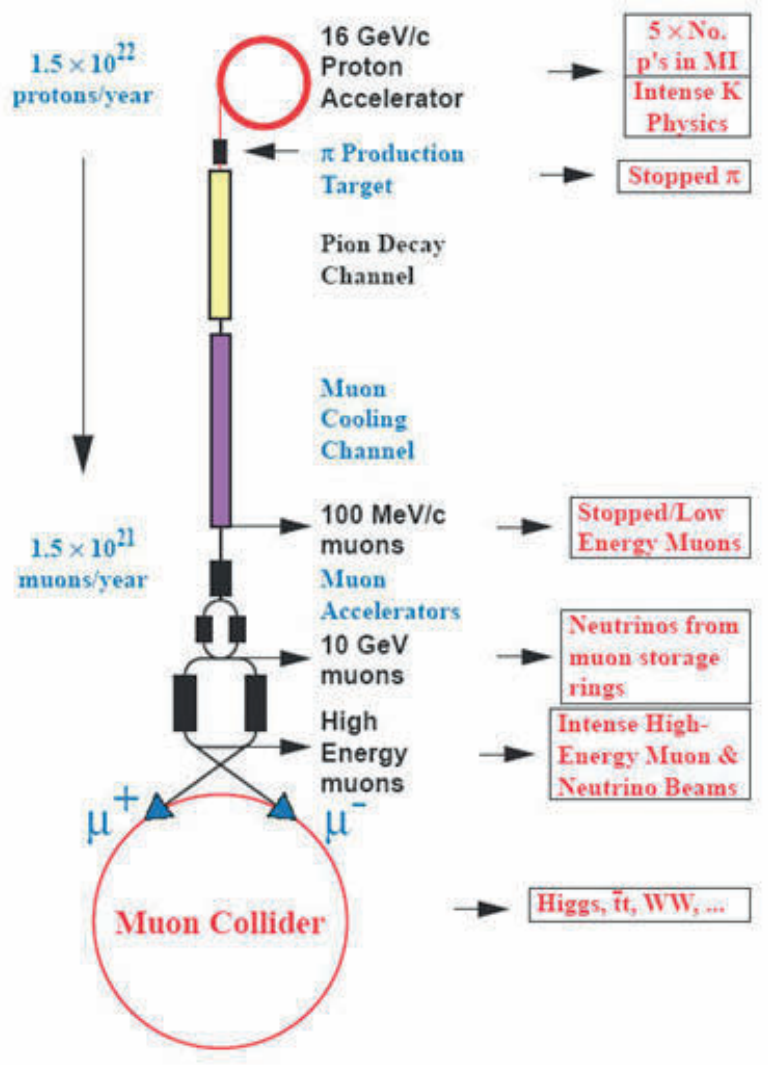

FIGURE 2. Schematic of a Muon Accelerator Facility.

ing. In particular, the 6D phase-space must be reduced by $\mathscr{O} 10^{6}$, which requires a longer and more complex cooling channel. Finally after the cooling channel, the muons are accelerated to the desired energy and injected into a decay (NF) or storage ring (MC). In a Neutrino Factory, the ring has long straight sections in which the neutrino beam is formed by the decaying muons. In a Muon Collider, positive and negative muons are injected in opposite directions and collide for about 1000 turns before the luminosity becomes marginalized due to muon decays.

\section{Low-energy muon physics}

From the description given above, it is clear that a muon accelerator facility that can produce (and manipulate) ultra-intense beams of muons can provide exciting opportunities for many physics programs: low-energy $\mu$ physics, neutrino oscillation physics and physics at the energy frontier. In this section I will elaborate briefly on what is possible at this facility with respect to low-energy $\mu$ physics.
One of the first physics programs that a muon accelerator facility could support would be sensitive tests of charged lepton flavor violation (cLFV), such as what could be explored with a $\mu \rightarrow$ e conversion experiment. In the Standard Model this process occurs via $v$ mixing, but the rate is well below what is experimentally accessible. The rate (or limit on the rate) of this process puts very stringent constraints on physics beyond the Standard Model. For example, supersymmetric models predict the rate to be $\mathscr{O} 10^{-15}$. The low-energy muon source of the muon accelerator facility provides a potential upgrade path for the next round of cLFV experiments currently being planned. Manipulation of the $6 \mathrm{D}$ emittance of the low-energy muon beam can produce a beam that has much higher stopping power in the target of the experiment. Fig. 3 shows the evolution of the momentum of a muon beam as it progresses down through sections of a helical cooling channel (HCC) [5]. This upgrade path could extend the sensitivity of these experiments by upwards of two orders of magnitude, exploring a mass reach to $4 \times 10^{4} \mathrm{TeV}$. 


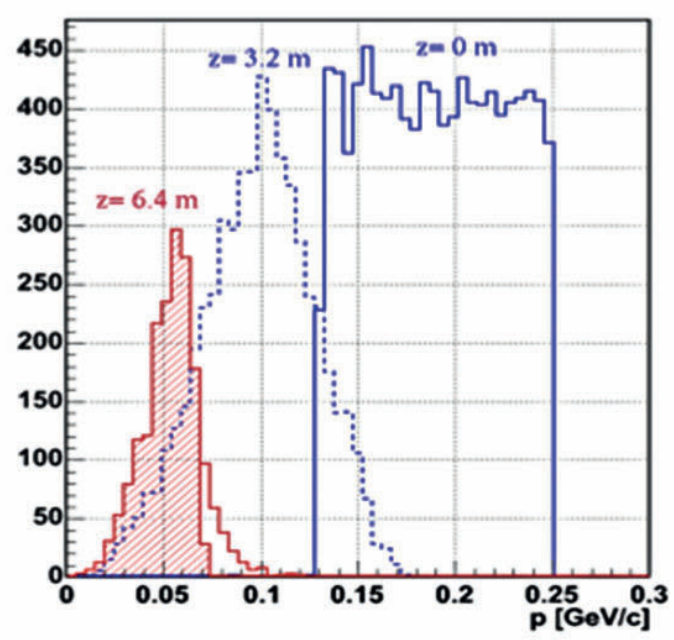

FIGURE 3. Evolution of the $\mu$ momentum spread from the start of a $\mathrm{HCC}(\mathrm{z}=0)$ through a series of sections ending at $\mathrm{z}=6.4 \mathrm{~m}$.

\section{The Energy Frontier}

As mentioned above, the Muon Collider could be the final step in the evolutionary process of the muon accelerator facility and it provides a very attractive possibility for studying the details of Terascale physics after the initial running of the LHC. The Muon Collider can study the same physics that electron-positron linear colliders address, but compared to these machines, a Muon Collider presents a very small footprint. It can easily fit on the Fermilab site (see Fig. 4), for example, and contains fewer complex components as a result. In addition, Muon Colliders may have a special role for precision measurements in that the machine potentially has a very small beam energy spread and thus allows for very precise energy scans. The beamstrahlung that exists at multi-TeV electron-positron linear colliders can limit their ultimate precision in this regard. This is illustrated in Fig. 5 [6].

\section{THE MUON ACCELERATOR PROGRAM}

Following over a decade of R\&D on Neutrino Factories and Muon Colliders, the US Neutrino Factory and Muon Collider Collaboration [7] and the Fermilab Muon Collider Task Force [8] jointly proposed a 7 year, roughly $\$ 120 \mathrm{M}$ R\&D program to the US Department of Energy. This proposal is aimed at completing a Design Feasibility Study (DFS) for a Muon Collider and, with international participation, a Reference Design Report (RDR) for a muon-based Neutrino Factory. The goal of the R\&D program is to provide the HEP community with detailed

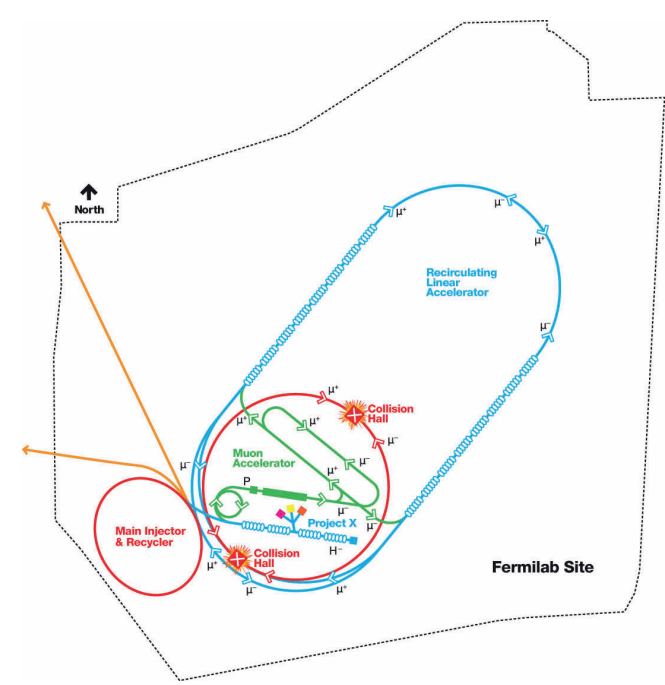

FIGURE 4. Muon Collider conceptual layout on the Fermilab site.

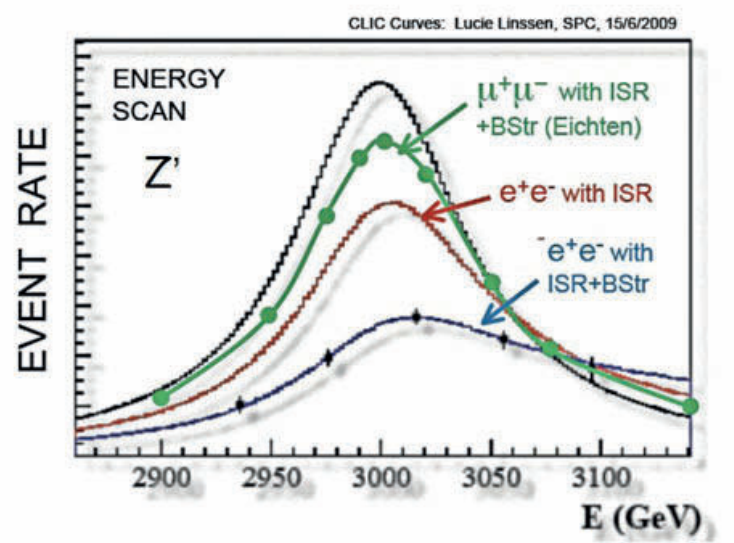

FIGURE 5. Representative energy-scan resolution for a 3 $\mathrm{TeV} \mathrm{Z}^{\prime}$ at representative machines.

information on future facilities based on intense beams of muons and give clear answers to questions regarding the expected capabilities and performance of these muon-based facilities. Providing defensible estimates for their cost is also to be part of the effort. This information, together with the physics insights gained from the next-generation neutrino and LHC experiments, will allow the HEP community to make well-informed decisions regarding the optimal choice for any new facility.

The proposal was reviewed in August of 2010 and was endorsed by an international review panel. The committee stated that the Muon Collider was a significant and important option for the world-wide High Energy Physics community and that a strategy that is based on a staged approach should be considered. 


\section{Program components}

The primary deliverables of MAP are enumerated below and described in some detail:

1. A Design Feasibility Study report (DFS) for a multi$\mathrm{TeV} \mathrm{MC}$ including an end-to-end simulation of the MC accelerator complex using demonstrated, or likely soon-to-be-demonstrated, technologies, an indicative cost range, and an identification of further technology R\&D that should be pursued to improve the performance and/or the cost effectiveness of the design.

2. Technology development and system tests that are needed to inform the Muon Collider DFS, and enable an initial down-selection of candidate technologies for the required ionization cooling and acceleration systems.

3. Contributions to the International Neutrino Factory Design Study (IDS-NF) to produce a Reference Design Report (RDR) for a NF by 2014. The emphasis of the proposed U.S. participation is on: a) design, simulation and cost estimates for those parts of the NF front-end that are (or could be) in common with a MC; b) studying how the evolving Fermilab proton source can be used for the Neutrino Factory RDR design; and c) studying how the resulting NF would fit on the Fermilab site

The two major deliverables (Muon Collider DFS and the Neutrino Factory RDR) are certainly paramount in that they will form the basis of the technical argument for these facilities. Looking at the individual sub-systems in these facilities, we can project where the Muon Accelerator Program will take the state-of-the-art with respect to these systems. Fig. 6 indicates where we believe each of the subsystems will be technically after this 7 year effort. In Fig. 6 level 7 (Muon Collider Proposal) represents the technical maturity (and understanding of the cost) needed in order to propose building the facility.

A separate physics and detector study (not part of the Muon Accelerator Program) will also be carried out in parallel with MAP. Initially, the physics case will be refined, benchmark processes with be defined, examination of the collision point environment will be done and basic detector performance criteria will be established so that the machine capabilities as a function of energy and luminosity of the collider can be determined. Close coordination between MAP and the Muon Collider physics and detector study will be facilitated by a Machine-Detector Interface working group within MAP. In parallel during the last 3 to 4 years of MAP, the physics and detector study will be devoted to detailed physics studies, including more complete detector simulations. In this period, comparisons with other possible facilities, such as CLIC

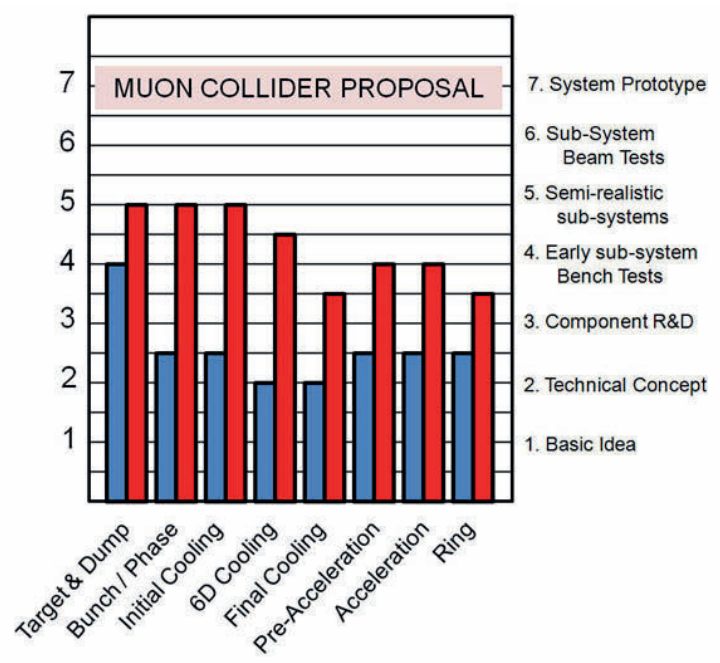

FIGURE 6. Sub-system technical progress after the 7 year MAP.

will also be carried out.

Work has already progressed on the understanding of the Muon Collider detector backgrounds [9]. From studies done over a decade ago, it was known that there were large backgrounds in the inner detectors from decay electrons. New studies that advance a shielding strategy of "electron-sweeping" upstream of the IP and optimization of a high- $\mathrm{Z}$ cone (a $10^{\circ} \mathrm{W}$ cone is used in the current simulations) near the IP have shown very good results. Fig. 8 shows the dose rate near the IP from a MARS [10] simulation of a $1.5 \mathrm{TeV}$ center-of-mass Muon Collider. The total dose is approximately $\frac{1}{2}$ that which would be seen at the LHC operating at a luminosity of $10^{34}$.

\section{Muon Collider Options}

Details regarding the basic design concepts for the Muon Collider are given in Fig. 7. As can be seen from this schematic, currently there are many options for the $6 \mathrm{D}$ muon ionization cooling and arguably the most crucial aspect of MAP is to efficiently carry out the initial phases of the cooling R\&D so that, as indicated in item 2 above, down-selection of candidate technologies can be accomplished early in the program.

\section{CONCLUSIONS}

A Muon Accelerator Program proposal has been approved by the US Department of Energy Office of High Energy Physics. The physics case for this type of facility has been well established and we now have the op- 




More R\&D needed to confirm viability and narrow the options

FIGURE 7. Sub-system options for the Muon Collider that are currently under study.

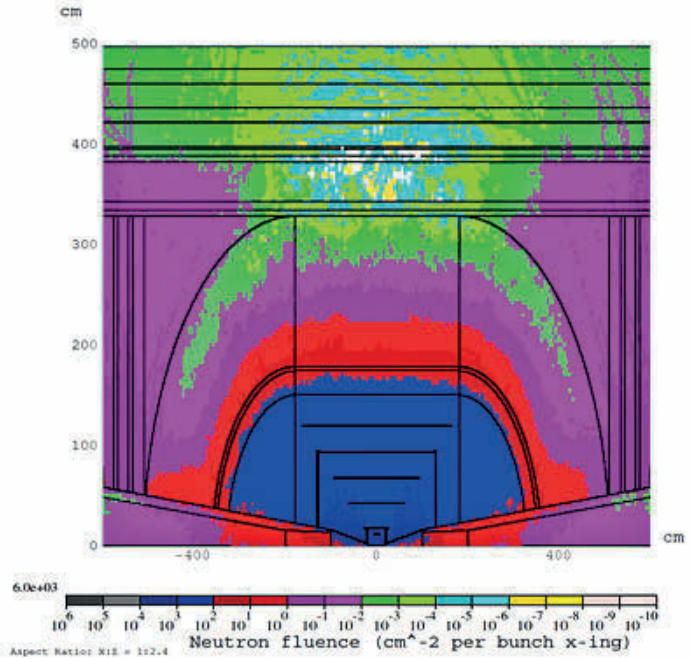

FIGURE 8. Total dose in the silicon detector at a $1.5 \mathrm{TeV}$ COM Muon Collider.

portunity to understand, in detail, its technical feasibility and cost. Delivering a Design Feasibility Study for a Muon Collider to the High Energy Physics community by around 2016 is the program's highest priority.

\section{ACKNOWLEDGMENTS}

I would like to thank all my colleagues in the Neutrino Factory and Muon Collider Collaboration and the Fermilab Muon Collider Task Force for all their support over the years and to acknowledge their amazing accomplishments over the past decade as we enter a new phase of our endeavor.

\section{REFERENCES}

1. J. Brau et al. [ILC Collaboration], arXiv:0712.1950 [physics.acc-ph].

2. J. P. Delahaye et al. [CLIC Study Group], in Proc. of the 20th Intl. Linac Conference LINAC 2000 ed. Alexander W. Chao, In the Proceedings of 20th International Linear Accelerator Conference (LINACO0), Monterey, California, 21-25 Aug 2000, pp MO201 [arXiv:physics/0008064].

3. E. Accomando et al. [CLIC Physics Working Group], arXiv:hep-ph/0412251.

4. M. Alsharo'a et al., (Muon Collider Collaboration), Phys. Rev. ST Accel. Beams, 6081001 (2003).

5. M. A. C. Cummings, R. P. Johnson, C. M. Ankenbrandt and K. Yonehara, In the Proceedings of Particle Accelerator Conference (PAC 07), Albuquerque, New Mexico, 25-29 Jun 2007, pp 2933.

6. E. Eichten, "Toward a MultiTeV Muon Collider: The Physics Case", Invited colloquium, Rutherford Appleton Laboratory, September 23, 2010.

7. See: http://www.cap.bnl.gov/mumu/

8. See: http://apc.fnal.gov/groups2/MCCC/

9. Y. I. Alexahin, E. Gianfelice-Wendt, V. V. Kashikhin, N. V. Mokhov, A. V. Zlobin and V. Y. Alexakhin [Neutrino Factory and Muon Collider Collaboration], In the Proceedings of 1st International Particle Accelerator Conference: IPAC'10, Kyoto, Japan, 23-28 May 2010, pp TUPEBO22.

10. N. V. Mokhov, "Status of MARS code," FERMILABCONF-03-053. 